1

2

3

\title{
Development of Computational Algorithms for Evaluating Option Prices Associated with Square-Root Volatility Processes
}

\author{
Hideyuki TAKADA ${ }^{*}$ Ushio SUMITA †and Hui JIN ${ }^{\ddagger}$
}

July 15, 2008

\begin{abstract}
The stochastic volatility model of Heston [6] has been accepted by many practitioners for pricing various financial derivatives, because of its capability to explain the smile curve of the implied volatility. While analytical results are available for pricing plain Vanilla European options based on the Heston model, there hardly exist any closed form solutions for exotic options. The purpose of this paper is to develop computational algorithms for evaluating the prices of such exotic options based on a bivariate birth-death approximation approach. Given the underlying price process $S_{t}$, the logarithmic process $U_{t}=\log S_{t}$ is first approximated by a birth-death process $B_{t}^{U}$ via moment matching. A second birth-death process $B_{t}^{V}$ is then constructed for approximating the stochastic volatility process $V_{t}$ through infinitesimal generator matching. Efficient numerical procedures are developed for capturing the dynamic behavior of $\left\{B_{t}^{U}, B_{t}^{V}\right\}$. Consequently, the prices of any exotic options based on the Heston model can be computed as long as such prices are expressed in terms of the joint distribution of $\left\{S_{t}, V_{t}\right\}$ and the associated first passage times. As an example, the prices of down-and-out call options are evaluated explicitly, demonstrating speed and fair accuracy of the proposed algorithms.
\end{abstract}

Keywords: stochastic volatility, barrier option, birth-death process, Meixner polynomials, uniformization procedure

\section{AMS 2000 Subject Classification: 60G15}

\section{Introduction}

In the area of financial engineering, one of the most important and influential theoretical results would be the celebrated Black-Scholes formula obtained by Black and Scholes [2] for

${ }^{*}$ Mizuho-DL Financial Technology Co.,Ltd. 1-1-3 Otemachi, Chiyoda-ku, Tokyo, Japan.

${ }^{\dagger}$ Graduate School of Systems and Information Engineering, University of Tsukuba, 1-1-1 Tennoudai, Tsukuba-City, Ibaraki, 305-8573, Japan.

${ }^{\ddagger}$ Graduate School of Systems and Information Engineering, University of Tsukuba, 1-1-1 Tennoudai, Tsukuba-City, Ibaraki, 305-8573, Japan. 
pricing options and other derivatives. The Black-Scholes model is built upon the following stochastic differential equation:

$$
d S_{t}=\mu S_{t} d t+\sigma S_{t} d W_{t} .
$$

Here, the price of the underlying financial asset follows the stochastic process $S_{t}$ with $\mu$ and $\sigma$ describing the trend and the volatility of $S_{t}$ respectively, and $W_{t}$ is the standard Brownian motion. When the structure of maturity, pay-off and strike price is specified, options and other derivatives defined on $S_{t}$ can be priced with no-arbitrage by solving (1.1) based on Ito's lemma and then applying the result to yield the desired pricing formula.

Since the development of the Black-Scholes model, many researchers have been trying to extend the original model so as to accommodate a variety of peculiar phenomena often observed in the market. For example, one of the major pitfalls of the original model can be found in that it can deal with only constant volatility $\sigma$. In reality, however, it is well known that the implied volatility ( which can be obtained by solving the Black-Scholes formula for $\sigma$ given the price, maturity and strike price) forms a smile curve as a function of the strike price, and it is impossible to explain this phenomenon under the assumption of the constant volatility.

The problem of how to deal with stochastic volatility is first solved by Hull and White [7], where $\sigma_{t}^{2}$ is assumed to follow a geometric Brownian motion. Subsequently, another extension is studied by Stein and Stein [11], where $\sigma_{t}^{2}$ is considered to be an OrnsteinUhlenbeck process. A more challenging stochastic volatility model is introduced by Heston [6] with a claim that the volatility of a price for many financial assets often follows a squareroot process. More specifically, let $S_{t}$ be the price of the underlying financial asset at time $t$ governed by the stochastic differential equation

$$
\frac{d S_{t}}{S_{t}}=r d t+\sigma \sqrt{V_{t}} d W_{t}^{(1)}
$$

and the volatility process $V_{t}$ itself is a diffusion process characterized by

$$
d V_{t}=\left(a-b V_{t}\right) d t+\delta \sqrt{V_{t}} d W_{t}^{(2)} .
$$

Here $\left\{W_{t}^{(1)}, W_{t}^{(2)}\right\}$ is a bivariate Brownian motion, and other parameters $r, \sigma, a, b$ and $\delta$ are constant.

In studying these extended models, the focus has been to solve the associated stochastic differential equation and then to derive a new pricing formula corresponding to the BlackScholes formula for plain Vanilla European options. In particular, the volatility smile mentioned before is constructed successfully based on the theoretical pricing formula in Heston [6]. Because of this reason, the square-root volatility process of Heston [6] has become quite prevalent among practitioners. To the best knowledge of the authors, however, no closed form solution is available for a variety of exotic options such as barrier options involving the Heston model. In other words, the study of the Heston model has been largely limited to the pricing of plain Vanilla European options. A rare exception is a recent paper by Gunter, Thomas and Uwe [5], where computational procedures have been developed for evaluating the prices of down-and-out call options. Their approach is based on discretization of the partial differential equation satisfied by the time-dependent reward function defined on the underlying stochastic processes. The discretization is necessary in both state and time, and the finite element methods are employed extensively for solving the resulting partial difference equations. Because of this, their approach may not be necessarily appropriate when 
The purpose of this paper is to develop computational algorithms for capturing the dynamic behavior of the bivariate process $\left\{S_{t}, V_{t}\right\}$ characterized by (1.2) and (1.3). The idea is to construct a bivariate birth-death process $\left\{B_{t}^{U}, B_{t}^{V}\right\}$ approximating $\left\{U_{t}, V_{t}\right\}$ where $U_{t}=\log S_{t}$. Prices of a variety of options including barrier options can then be readily computed with speed and accuracy. Our approach may be more systematic and can tolerate repeated computations better than the approach of Gunter, Thomas and Uwe [5] because of the following reasons.

(1) In Gunter, Thomas and Uwe [5], the discretized partial difference equation is developed directly for each reward process. Accordingly, the price computation has to be repeated almost independently for each option and for each parameter set. In our approach, the dynamic behavior of $\left\{S_{t}, V_{t}\right\}$ is first captured. The computational results for the dynamic behavior are common in evaluating any option price and can be used repeatedly. Furthermore, changes due to different parameter values are restricted entirely to the change of two birth-death stochastic matrices and the whole algorithmic procedures remain intact.

(2) In Gunter, Thomas and Uwe [5], both the state space and time have to be discretized. This means that, given a discretized time interval $[t, t+\Delta]$, the value of the underlying financial asset can move only in a lattice continuous manner. In contrast, our approach requires to descretize only the state space, and the approximating birth-death process can move from any state to any other state with positive probability in a time interval $[t, t+\Delta]$. This feature may be advantageous when the volatility level is high.

The structure of this paper is as follows. In Section 2, the square-root volatility model of Heston [6] is formally introduced. The time dependent conditional transition probability structure of $U_{t}=\log S_{t}$ is described explicitly. Section 3 discusses the birth-death process approximation of $U_{t}$ where $B_{t}^{U}$ is constructed explicitly via moment matching. In Section 4 , the birth-death process $B_{t}^{V}$ is constructed similarly but based on infinitesimal generator matching, approximating the square-root process $V_{t}$. The transition probability structure of $\left\{B_{t}^{U}, B_{t}^{V}\right\}$ is described in Section 5, and the volatility smile is evaluated as a function of both the strike price and maturity in Section 6. Finally, numerical results are presented in Section 7, demonstrating the computational efficiency for pricing down-and-call options defined on $S_{t}$. 


\section{Square Root Process for Stock Price Volatility}

In this section, we consider a stochastic process defined as the logarithm of $S_{t}$ associated with the stochastic volatility model of Heston [6] characterized by (1.2) and (1.3). We restrict ourselves to the case that $W_{t}^{(1)}$ and $W_{t}^{(2)}$ are independent. Furthermore, the condition $2 a>\delta^{2}$ is imposed so that the square-root process $V_{t}$ remains positive provided that the initial value is positive, see e.g. Lamberton and Lapeyre [10]. Given $V_{t}=v$, by using Ito's Lemma, Equation (1.2) can be rewritten as

$$
\left.d\left(\log S_{t}\right)\right|_{V_{t}=v}=\left(r-\frac{\sigma^{2}}{2} v\right) d t+\sigma \sqrt{v} d W_{t}^{(1)} .
$$

With $S_{0}=s_{0}$, this then leads to

$$
\left.\log S_{t}\right|_{V_{t}=v}=\left(r-\frac{\sigma^{2}}{2} v\right) t+\sigma \sqrt{v}\left(W_{t}^{(1)}-W_{0}^{(1)}\right)+\log s_{0} .
$$

For notational convenience, let $U_{t}=\left.\log S_{t}\right|_{V_{t}=v}$. Then (2.2) can be rewritten as

$$
U_{t}=\left(r-\frac{\sigma^{2}}{2} v\right) t+\sigma \sqrt{v}\left(W_{t}^{(1)}-W_{0}^{(1)}\right)+u_{0}
$$

where $U_{0}=u_{0}=\log s_{0}$. Since $W_{t}^{(1)}-W_{0}^{(1)}$ follows the normal distribution $N(0, t)$, the distribution of $U_{t}$ for each $t \geq 0$ is also normal with its mean and variance given by

$$
\begin{cases}E\left[U_{t} \mid U_{0}=u_{0}\right] & =u_{0}+\left(r-\frac{\sigma^{2}}{2} v\right) t \\ \operatorname{Var}\left[U_{t} \mid U_{0}=u_{0}\right] & =\sigma^{2} v t\end{cases}
$$

Accordingly its conditional density function $g\left(u_{0}, x, t\right)=\frac{d}{d x} P\left[U_{t} \leq x \mid U_{0}=u_{0}\right]$ is given by

$$
g\left(u_{0}, x, t\right)=\frac{1}{\sqrt{2 \pi \sigma^{2} v t}} \exp \left[-\frac{\left\{x-\left(u_{0}+\left(r-\frac{\sigma^{2}}{2} v\right) t\right)\right\}^{2}}{2 \sigma^{2} v t}\right] .
$$

For tail probabilities of $g\left(u_{0}, x, t\right)$ with respect to $x$, we define

$$
\bar{G}\left(u_{0}, x, t\right)=\int_{x}^{\infty} g\left(u_{0}, y, t\right) d y \text {. }
$$

Values of $\bar{G}\left(u_{0}, x, t\right)$ can be computed with speed and accuracy, e.g. using the Laguerre transform where 12 digit accuracy is achieved for such computations. The reader is referred to Sumita [12], and Sumita and Kijima [13, 14] for further details.

\section{Birth-Death Process Approximation of $U_{t}=\log S_{t}$ via Moment Matching}

In order to develop computational procedures for evaluating the prices of European options and other exotic options defined on $S_{t}$ of (1.2), we attempt to approximate the bivariate process $\left\{U_{t}, V_{t}\right\}$ by a Markov chain defined on another Markov chain. For this purpose, we first approximate the stochastic process $U_{t}$ by a birth-death process $B_{t}^{U}$ via a moment matching method. Let $R_{U}=\left[u_{\text {Begin }}, u_{\text {End }}\right]$ be a subset of the state space of $U_{t}$ such that 
$P\left[U_{t} \in R_{U}\right]$ is almost one. More formally, given a sufficiently small $\epsilon>0$, there exist $u_{\text {Begin }}$ and $u_{E n d}$ such that

$$
1-P\left[U_{t} \in R_{U}\right]<\epsilon \text { for all } t \geq 0 .
$$

A discrete state space $\mathcal{N}=\{0,1,2, \cdots, N\}$ is then introduced where $u_{0}=u_{\text {Begin }}, u_{N}=u_{\text {End }}$, and

$$
h_{U}=u_{i}-u_{i-1}, i=1,2, \cdots, N \text {. }
$$

Let $R_{V}=\left[v_{B e g i n}, v_{E n d}\right]$ be defined similarly for the process $V_{t}$ with $\mathcal{J}=\{0,1, \cdots, J\}$ where $v_{0}=v_{B e g i n}, v_{J}=v_{E n d}$,

$$
1-P\left[V_{t} \in R_{V}\right]<\epsilon \text { for all } t \geq 0,
$$

and

$$
h_{V}=v_{j}-v_{j-1}, j=1,2, \cdots, J .
$$

Given $V_{t}=v_{j}, j \in \mathcal{J}$, we now approximate the process $U_{t}$ by a birth-death process $B_{t}^{U}$ defined on $\mathcal{N}$. Suppose that the approximating birth-death process is governed by a hazard rate matrix

$$
\underline{\nu}_{U}(j)=\left(\begin{array}{cccccccc}
0 & \nu_{0}^{+}(j) & 0 & 0 & \ldots & 0 & 0 & 0 \\
\nu_{1}^{-}(j) & 0 & \nu_{1}^{+}(j) & 0 & \ldots & 0 & 0 & 0 \\
0 & \nu_{2}^{-}(j) & 0 & \nu_{2}^{+}(j) & \ldots & 0 & 0 & 0 \\
\vdots & \vdots & \vdots & \vdots & \ddots & \vdots & \vdots & \vdots \\
0 & 0 & 0 & 0 & \ldots & 0 & \nu_{N-2}^{+}(j) & 0 \\
0 & 0 & 0 & 0 & \ldots & \nu_{N-1}^{-}(j) & 0 & \nu_{N-1}^{+}(j) \\
0 & 0 & 0 & 0 & \ldots & 0 & \nu_{N}^{-}(j) & 0
\end{array}\right),
$$

where $\nu_{i}^{+}(j)$ and $\nu_{i}^{-}(j)$ denote the upward transition rate and the downward transiton rate at state $i \in \mathcal{N}$ respectively. The idea behind the moment matching method proposed here is to determine $\underline{\underline{\nu}}_{U}(j)$ in such a way that the following equation holds true.

$$
\log E\left[e^{-\theta d U_{t}} \mid U_{t}=u_{i}\right]=\log E\left[e^{-\theta d B_{t}} \mid B_{t}^{U}=i\right]+o\left(\theta^{2}\right) d t
$$

The following theorem can then be shown.

Theorem 3.1 Given $V_{t}=v_{j}, j \in \mathcal{J}$, let $B_{t}^{U}$ be a birth-death process on $\mathcal{N}$ governed by $\underline{\underline{\nu}}_{U}(j)$ of (3.5) satisfying (3.6). One then has $\nu_{0}^{+}(j)=\nu_{N}^{-}(j)=\frac{\sigma^{2} v_{j}}{h_{U}^{2}}$ and, for $i \in \mathcal{N} \backslash\{0, N\}$,

$$
\left\{\begin{array}{l}
\nu_{i}^{+}(j)=\frac{\sigma^{2} v_{j}+h_{U}\left(r-\frac{\sigma^{2} v_{j}}{2}\right)}{2 h_{U}^{2}}, \\
\nu_{i}^{-}(j)=\frac{\sigma^{2} v_{j}-h_{U}\left(r-\frac{\sigma^{2} v_{j}}{2}\right)}{2 h_{U}^{2}} .
\end{array}\right.
$$

Proof. Given $V_{t}=v_{j}$, we first note that

$$
\begin{aligned}
\log E\left[e^{-\theta d U_{t}} \mid U_{t}=u_{i}\right] & =-E\left[\theta d U_{t}\right]+\frac{1}{2} \operatorname{Var}\left[\theta d U_{t}\right]+o\left(\theta^{2}\right) d t \\
& =-\theta\left(r-\frac{\sigma^{2}}{2} v_{j}\right) d t+\frac{1}{2} \theta^{2} \sigma^{2} v_{j} d t+o\left(\theta^{2}\right) d t .
\end{aligned}
$$


On the other hand, under (3.6), one has

$$
\begin{aligned}
& \log E\left[e^{-\theta\left(u_{t+\Delta t}-u_{t}\right)} \mid U_{t}=u_{i}\right] \\
= & \log \left[\left\{1-\Delta t\left(\nu_{i}^{-}(j)+\nu_{i}^{+}(j)\right)\right\}+\nu_{i}^{-}(j) \Delta t e^{-\theta\left(u_{i-1}-u_{i}\right)}+\nu_{i}^{+}(j) \Delta t e^{-\theta\left(u_{i+1}-u_{i}\right)}+o(\Delta t)\right] \\
& +o\left(\theta^{2}\right) \Delta t .
\end{aligned}
$$

Since $h_{U}=u_{i}-u_{i-1}$, using the Talyor expansions of $e^{-\theta h_{U}}$ and $e^{\theta h_{U}}$, the above equation leads to

$$
\begin{aligned}
& \log \left[1-\left\{\nu_{i}^{-}(j)+\nu_{i}^{+}(j)\right\} \Delta t+\nu_{i}^{-}(j) \Delta t\left\{1+\theta h_{U}+\frac{1}{2} \theta^{2} h_{U}^{2}+o\left(\theta^{2}\right)\right\}\right. \\
& \left.+\nu_{i}^{+}(j) \Delta t\left\{1-\theta h_{U}+\frac{1}{2} \theta^{2} h_{U}^{2}+o\left(\theta^{2}\right)\right\}+o(\Delta t)\right]+o\left(\theta^{2}\right) \Delta t \\
= & \log \left[1+\Delta t\left\{-\left(\nu_{i}^{-}(j)-\nu_{i}^{+}(j)\right) h_{U} \theta+\left(\nu_{i}^{-}(j)+\nu_{i}^{+}(j)\right) \frac{1}{2} h_{U}^{2} \theta^{2}+o\left(\theta^{2}\right)\right\}+o(\Delta t)\right]+o\left(\theta^{2}\right) \Delta t .
\end{aligned}
$$

By employing the identity $\log (1+x)=\sum_{k=1}^{\infty}(-1)^{k-1} \frac{x^{k}}{k}$, it follows that

$$
\log E\left[e^{-\theta\left(u_{t+\Delta t}-u_{t}\right)} \mid U_{t}=u_{i}\right]=\Delta t\left\{-\left(\nu_{i}^{-}(j)-\nu_{i}^{+}(j)\right) h_{U} \theta+\left(\nu_{i}^{-}(j)+\nu_{i}^{+}(j)\right) \frac{1}{2} h_{U}^{2} \theta^{2}+o\left(\theta^{2}\right)\right\}+o(\Delta t),
$$

or equivalently

$$
\begin{gathered}
\log E\left[e^{-\theta d U_{t}} \mid U_{t}=u_{i}\right]=\lim _{\Delta t \rightarrow 0} \frac{\log E\left[e^{-\theta\left(u_{t+\Delta t}-u_{t}\right)} \mid U_{t}=u_{i}\right]}{\Delta t} d t \\
=-\left\{\nu_{i}^{-}(j)-\nu_{i}^{+}(j)\right\} h_{U} \theta d t+\left\{\nu_{i}^{-}(j)+\nu_{i}^{+}(j)\right\} \frac{1}{2} h_{U}^{2} \theta^{2} d t+o\left(\theta^{2}\right) d t,
\end{gathered}
$$

where $\nu_{0}^{-}(j)=\nu_{N}^{+}(j)=0$. Comparing (3.9) with (3.10), one then has, for $i \in \mathcal{N} \backslash\{0, N\}$,

$$
\begin{cases}r-\frac{\sigma^{2}}{2} v_{j} & =h_{U} \nu_{i}^{-}(j)-h_{U} \nu_{i}^{+}(j), \\ \sigma^{2} v_{j} & =h_{U}^{2} \nu_{i}^{-}(j)+h_{U}^{2} \nu_{i}^{+}(j),\end{cases}
$$

and $\nu_{0}^{+}(j)=\nu_{N}^{-}(j)=\frac{\sigma^{2} v_{j}}{h_{U}^{2}}$. The theorem now follows by solving $(3.10)$ for $\nu_{i}^{-}(j)$ and $\nu_{i}^{+}(j)$.

It should be noted that

$$
\nu_{i}^{+}(j)+\nu_{i}^{-}(j)=\frac{\sigma^{2} v_{j}}{h_{U}^{2}},
$$

so that $\nu_{i}^{+}(j)+\nu_{i}^{-}(j)$ is independent of $i$ and constant when $\sigma, v_{j}$ and $h_{U}$ are determined.

Given $U_{0}=u_{0}$, the tail probability $\bar{G}\left(u_{0}, x, t\right)$ of $U_{t}$ at $x$ is defined in (2.6) and can be computed via the Laguerre transform with 12 digit accuracy. When $U_{t}$ is approximated by the birth-death process $B_{t}^{U}$, this tail probability is approximated accordingly by $\bar{G}\left(i_{m}, i_{n}, t\right), i_{m}, i_{n} \in \mathcal{N}$, describing the sum of the transition probabilities of $B_{t}^{U}$ from $i_{m}$ to $i$ for $i_{n} \leq i \leq N$ at time t, where $i_{m}$ and $i_{n}$ in $\mathcal{N}$ correspond to the closest points to $u_{0}$ and $x$ respectively. One then expects that

$$
\bar{G}\left(i_{m}, i_{n}, t\right) \rightarrow \bar{G}\left(u_{0}, x, t\right) \text { as } h_{U} \rightarrow 0 .
$$

In Table 3.1 below, numerical results are presented for demonstrating the accuracy of the birth-death approximation of $U_{t}$ by $B_{t}^{U}$ based on (3.12). For necessary parameter values, 


\section{Birth-Death Process Approximation of $V_{t}$ via Infinites- imal Generator Matching}

For approximating the bivariate process $\left\{U_{t}, V_{t}\right\}$ by a Markov chain defined on another Markov chain, the second step is to find a birth-death process $B_{t}^{V}$ representing a discretized version of $V_{t}$. Unlike the previous discussion concerning $U_{t}$, however, the moment matching approach of Section 2 is not applicable here simply because $E\left[e^{-\theta d V_{t}} \mid V_{t}=v_{j}\right]$ is not readily 
available. As an alternative approach, following Albanese and Kuznetsov [1], the birth-death process $B_{t}^{V}$ is constructed in such a way that the second order difference equations satisfied by the orthogonal polynomials associated with $B_{t}^{V}$ parallel the second order differential equations associated with the square-root process $V_{t}$.

Let $f\left(x_{0}, x, t\right)$ be the conditional density function of the square-root process $V_{t}$ defined in (1.3), i.e.

$$
f\left(x_{0}, x, t\right)=\frac{d}{d x} P\left[V_{t} \leq x \mid V_{0}=x_{0}\right] .
$$

For $R^{+}=(0, \infty)$, let

$$
L=\left\{q: R^{+} \rightarrow R^{+} \mid \int_{0}^{\infty} f\left(x_{0}, x, t\right) q(x) d x<\infty \text { for any } t \geq 0, x_{0} \geq 0\right\}
$$

and define an operator $\mathcal{P}_{t}: L \times\{t\} \rightarrow R^{+}$by

$$
\left(\mathcal{P}_{t} q\right)_{\left(x_{0}\right)}=E_{x_{0}}\left[q\left(V_{t}\right)\right]=\int_{0}^{\infty} f\left(x_{0}, x, t\right) q(x) d x .
$$

Given $a, b$ and $\delta$ associated with $V_{t}$ in (1.3), let $\mathcal{L}$ be an operator defined by

$$
\mathcal{L} \stackrel{\text { def. }}{=}(a-b x) \frac{d}{d x}+\frac{\delta^{2} x}{2} \frac{d^{2}}{d x^{2}} .
$$

It is then known, see e.g. Schoutens [15], that the operators $\mathcal{P}_{t}$ and $\mathcal{L}$ are related to each other by the Kolmogorov forward equation

$$
\frac{\partial}{\partial t}\left(\mathcal{P}_{t} q\right)_{\left(x_{0}\right)}=\mathcal{L}\left(\mathcal{P}_{t} q\right)_{\left(x_{0}\right)}=P_{t}(\mathcal{L} q)_{\left(x_{0}\right)},
$$

or equivalently

$$
\frac{\partial}{\partial t} \int_{0}^{\infty} f\left(x_{0}, x, t\right) q(x) d x=\int_{0}^{\infty} f\left(x_{0}, x, t\right) \mathcal{L} q(x) d x .
$$

Because of the commutativity of $\mathcal{L}$ and $\mathcal{P}_{t}$ in (4.5), the operator $\mathcal{P}_{t}$ may be written symbolically as

$$
\mathcal{P}_{t}=e^{t \mathcal{L}}=\sum_{k=0}^{\infty} \frac{t^{k} \mathcal{L}^{k}}{k !}
$$

where $\mathcal{L}^{0}$ is the identity operator and $\mathcal{L}^{k}$ for $k \geq 1$ means that the operator $\mathcal{L}$ is applied $k$ times repeatedly. In this sense, the operator $\mathcal{L}$ can be interpreted as the infinitesimal generator of the square-root process $V_{t}$.

In order to find the birth-death process $B_{t}^{V}$ representing a discrete version of $V_{t}$, our approach is to find an infinitesimal generator matrix $\underline{\underline{Q}}$ of a birth-death process which parallels the operator $\mathcal{L}$. For this purpose, following Albanese and Kuzentsov [1], we exploit the fact that a discrete operator generated by Meixner polynomials associated with a linear birthdeath process is similar to $\mathcal{L}$. More specifically, a linear birth-death process is considered where upward transition rates $\lambda_{j}(j \geq 0)$ and downward transtion rates $\mu_{j}(j \geq 1)$ are given by

$$
\lambda_{j}=\beta c+j c, \quad j \geq 0 ; \quad \mu_{j}=j, \quad j \geq 1,
$$


with $\beta>0$ and $0<c<1$. It is known, see Karlin and McGregor [8], that the set of orthogonal polynomials, called Meixner polynomials, associated with this birth-death process can be generated by the recursive formula

$$
M_{n+1}(x ; \beta, c)=\frac{1}{c(n+\beta)}\left[\{(c-1) x+n+(n+\beta) c\} M_{n}(x ; \beta, c)-n M_{n-1}(x ; \beta, c)\right]
$$

for $n \geq 1$ starting with $M_{0}(x ; \beta, c)=1$ and $M_{1}(x ; \beta, c)=\{(c-1) x+n+(n+\beta) c\} /\{c(n+\beta)\}$.

We recall that, given a sufficiently small $\epsilon>0, R_{V}=\left[v_{B e g i n}, v_{E n d}\right]$ is determined so that (3.3) is satisfied. By discretizing $R_{V}$ with $J$ equal intervals, one has $h_{V}$ as given in (3.4). Corresponding to $L$ in $(4.2)$, let

$$
\widehat{L}=\left\{\underline{\hat{q}}=[\hat{q}(0), \cdots, \hat{q}(J)] \mid \hat{q}(j)=q\left(v_{j}\right), q \in L, j \in \mathcal{J}\right\},
$$

where $\mathcal{J}=\{0,1, \cdots, J\}$. In parallel with the continuum operator $\mathcal{L}: L \rightarrow R$ given in (4.4), a discrete operator $\widehat{\mathcal{L}}: \widehat{L} \rightarrow R$ is introduced as

$$
\widehat{\mathcal{L}} \stackrel{\text { def. }}{=} M \times\left\{(\beta c-(1-c) j) \nabla+j \nabla^{2}\right\}, j \in\{0,1, \cdots, J\},
$$

where $M$ is a positive constant and, for a vector $\underline{\hat{q}}=[\hat{q}(0), \cdots, \hat{q}(J)], \nabla$ and $\nabla^{2}$ are defined in the following manner. We first define

$$
\nabla^{+} \hat{q}(j)=\hat{q}(j+1)-\hat{q}(j) ; \nabla^{-} \hat{q}(j)=\hat{q}(j)-\hat{q}(j-1),
$$

where $\hat{q}(j)=0$ whenever $j \notin \mathcal{J}$. Based on (4.12), $\nabla$ and $\nabla^{2}$ are now given as

$$
\left\{\begin{array}{l}
\nabla \hat{q}(j)=\nabla^{+} \hat{q}(j)=\hat{q}(j+1)-\hat{q}(j) \\
\nabla^{2} \hat{q}(j)=\nabla^{+} \nabla^{-} \hat{q}(j)=\hat{q}(j+1)+\hat{q}(j-1)-2 \hat{q}(j) .
\end{array}\right.
$$

By comparing $\mathcal{L}\left[q\left(v_{j}\right)\right]$ with $\widehat{\mathcal{L}}[\hat{q}(j)]$, and matching the coefficients of $d / d x$ and $(d / d x)^{2}$ with those of $\nabla$ and $\nabla^{2}$, one finds that

$$
\left\{\begin{array}{l}
M=\frac{\delta^{2}}{2 h_{V}} \\
c=1-\frac{2 h_{V} b}{\delta^{2}} \\
\beta=\frac{2 a}{c \delta^{2}} .
\end{array}\right.
$$

Consequently, the square-root process $V_{t}$ can be approximated by the linear birth-death process $B_{t}^{V}$ defined on $\mathcal{J}=\{0,1, \cdots, J\}$ governed by upward transition rates $M \lambda_{j}(j \geq 0)$ and downward transition rates $M \mu_{j}(j \geq 1)$ where $\lambda_{j}$ and $\mu_{j}$ are as given in (4.8) with $M, c$ and $\beta$ as defined in (4.14).

\section{Transition Probability Structure of $\left\{B_{t}^{U}, B_{t}^{V}\right\}$}

Based on the previous discussions, one now sees that the bivariate birth-death process $\left\{B_{t}^{U}, B_{t}^{V}\right\}$ approximates the bivariate process $\left\{U_{t}, V_{t}\right\}$, where the transition probability structure of $\left\{B_{t}^{U}, B_{t}^{V}\right\}$ is characterized by the following transition rate matrix $\underline{\underline{\nu}}$ :

$$
\underline{\underline{\nu}}=\left(\begin{array}{ccccccccccc}
\underline{\nu}_{U}(0) & \lambda_{0} \underline{\underline{I}} & 0 & \ldots & 0 & 0 & 0 & \ldots & 0 & 0 & 0 \\
\mu_{1} \underline{\underline{I}} & \underline{\underline{\nu}}_{U}(1) & \lambda_{1} \underline{\underline{I}} & \ldots & 0 & 0 & 0 & \ldots & 0 & 0 & 0 \\
\vdots & \vdots & \vdots & \ddots & \ddots & \ddots & \ddots & \ddots & \vdots & \vdots & \vdots \\
0 & 0 & 0 & \ldots & \mu_{j} \underline{\underline{I}} & \underline{\underline{\nu}}_{U}(j) & \lambda_{j} \underline{\underline{I}} & \ldots & 0 & 0 & 0 \\
\vdots & \vdots & \vdots & \ddots & \ddots & \ddots & \ddots & \ddots & \vdots & \vdots & \vdots \\
0 & 0 & 0 & \ldots & 0 & 0 & 0 & \ldots & \mu_{J-1} \underline{\underline{I}} & \underline{\underline{\nu}}_{U}(J-1) & \lambda_{J-1} \underline{\underline{I}} \\
0 & 0 & 0 & \ldots & 0 & 0 & 0 & \ldots & 0 & \mu_{J} \underline{\underline{I}} & \underline{\underline{\nu}}_{U}(J)
\end{array}\right)
$$


Here $\lambda_{j}$ and $\mu_{j}$ are given in (4.8) with $\underline{\underline{\nu}}_{U}(j)$ as in (3.5).

Let $\underline{\underline{P}}(t)=\left[p_{\left(i_{m}, j_{k}\right),\left(i_{n}, j_{l}\right)}(t)\right], i_{m}, i_{n} \in \mathcal{N} ; j_{l}, j_{k} \in \mathcal{J}$ be the transiton probability matrix of $\left\{B_{t}^{U}, B_{t}^{V}\right\}$. In what follows, we show how to evaluate $\underline{\underline{P}}(t)$ based on the uniformization procedure of Keilson [9]. Given a matrix $\underline{\underline{l}}=\left[l_{i j}\right]$, let $l_{i}=\sum_{j} l_{i j}$ and define the diagonal matrix $\underline{\underline{l}}_{D}$ by $\underline{\underline{l}}_{D}=\left[\delta_{\{i=j\}} l_{i}\right]$, where $\delta_{\{P\}}=1$ if statement $P$ holds true and $\delta_{\{P\}}=0$ otherwise. For $\underline{\underline{\nu}}$ of $(5.1)$, the block diagonal matrices of $\underline{\underline{\nu}}_{D}$ are then given by

$$
\begin{cases}\underline{\underline{\nu}}_{U: D}(0)+\lambda_{0} \underline{\underline{I}} & j=0, \\ \underline{\underline{\nu}}_{U: D}(j)+\left(\mu_{j}+\lambda_{j}\right) \underline{\underline{I}} & j=1,2, \cdots, J-1, \\ \underline{\underline{\nu}}_{U: D}(J)+\mu_{J} \underline{\underline{I}} & j=J .\end{cases}
$$

We note that $\underline{\underline{\nu}}_{U: D}(j)$ is the diagonal matrix generated by the row sums of $\underline{\underline{\nu}}_{U}(j)$. From the Kolmogorov forward equation $\frac{d}{d t} \underline{\underline{P}}(t)=\underline{\underline{P}}(t) \underline{\underline{Q}}$, one has

$$
\underline{\underline{P}}(t)=e \stackrel{t Q}{=} ; \underline{\underline{Q}}=-\underline{\underline{\nu}}_{D}+\underline{\underline{\nu}} \text {. }
$$

Let $\nu$ be a positive number which is larger than or equal to the maximum diagonal element of $\underline{\underline{\nu}}_{D}$ and define a matrix $\underline{\underline{a}}_{\nu}$ by

$$
\underline{\underline{a}} \nu \stackrel{\text { def }}{=} \underline{\underline{I}}-\frac{1}{\nu} \underline{\underline{\nu}}_{D}+\frac{1}{\nu} \underline{\underline{\nu}}
$$

It can be readily seen that $\underline{\underline{a}}_{\nu}$ is a stochastic matrix. From (5.3) and (5.4), one has $\underline{\underline{a}}_{\nu}=\underline{\underline{I}}+\frac{1}{\nu} \underline{\underline{Q}}$ so that $\underline{\underline{Q}}=-\nu\left[\underline{\underline{I}}-\underline{\underline{a}}_{\nu}\right]$. Substituting this into (5.3), it then follows that

$$
\underline{\underline{P}}(t)=e^{-\nu t\left[\underline{\underline{I}}_{-}^{-} \underline{\underline{a}}_{\nu}\right]}=\sum_{k=0}^{\infty} e^{-\nu t} \frac{(\nu t)^{k}}{k !} \underline{\underline{a}}_{\nu}^{k}
$$

where $\underline{\underline{a}}_{\nu}^{0}=\underline{\underline{I}}$. Since the series representation of (5.5) involves only nonnegative numbers, the computational procedure is stable and a fairly large size of matrices can be incorporated with speed and high accuracy. In addition, for the application of this paper, it is possible to exploit the underlying block tri-diagonal structure for reducing the computational burden substantially, as we will see next.

Let $\underline{\underline{a}}_{U: \nu}(j)$ be defined by

$$
\underline{\underline{a}}_{U: \nu}(j)=\underline{\underline{I}}-\frac{1}{\nu} \underline{\underline{\nu}}_{U: D}(j)+\frac{1}{\nu} \underline{\underline{\nu}}_{U}(j), j=0,1, \cdots, J .
$$

Furthermore, we also introduce

$$
\underline{\underline{\hat{a}}}_{U: \nu}(j)= \begin{cases}\underline{\underline{a}}_{U: \nu}(0)-\frac{\lambda_{0}}{\nu} \underline{\underline{\underline{I}}} & j=0, \\ \underline{\underline{a}}_{U: \nu}(j)-\frac{\mu_{j}+\lambda_{j}}{\nu} \underline{I} & j=1,2, \cdots, J-1, \\ \underline{\underline{a}}_{U: \nu}(J)-\frac{\mu_{J}}{\nu} \underline{\underline{I}} & j=J .\end{cases}
$$

The stochastic matrix $\underline{a}_{\nu}$ of (5.4) for the application of this paper can then be given as

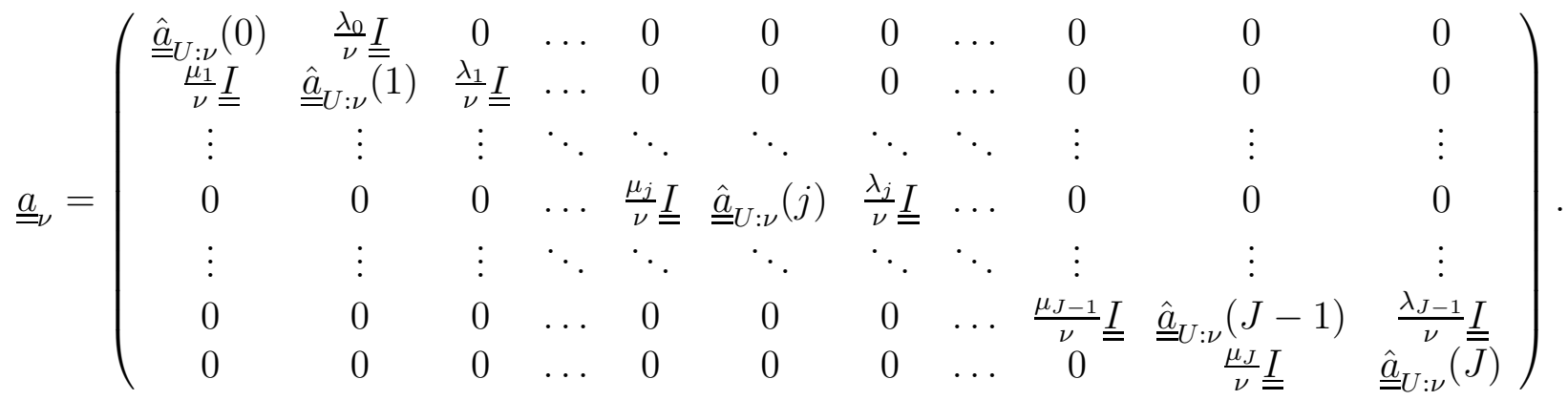


For evaluating the implied volatility and exotic option prices associated with (1.2) and (1.3), what we actually need is the state probability vector given an initial state probability vector, not the transition probability matrix itself. In this regard, we define the state probability vector $\underline{p}^{\top}(t)$ by

$$
\underline{p}^{\top}(t)=\left[\underline{p}_{0}^{\top}(t), \cdots, \underline{p}_{j}^{\top}(t), \cdots, \underline{p}_{J}^{\top}(t)\right]
$$

where $\underline{p}_{j}^{\top}(t)=\left[p_{(0, j)}(t), \cdots, p_{(N, j)}(t)\right]$ and

$$
p_{(i, j)}(t)=P\left[U_{t}=i, V_{t}=j \mid \underline{p}^{\top}(0)\right] .
$$

According to $(5.5), \underline{p}^{\top}(t)=\underline{p}^{\top}(0) \times \underline{\underline{P}}(t)$ can be evaluated by

$$
\underline{p}^{\top}(t)=\sum_{k=0}^{\infty} e^{-\nu t} \frac{(\nu t)^{k}}{k !} \underbrace{p^{\top}(0) \underline{\underline{a}}^{k}}_{A},
$$

where the underbraced part $A$ can be evaluated by

$$
\left\{\begin{array}{lll}
\underline{p}_{0}^{\top}(t)=\underline{p}_{0}^{\top}(0) \underline{\hat{a}}_{U: D}(0)+\frac{\mu_{1}}{\nu} \underline{p}_{1}^{\top}(0) & & j=0, \\
\underline{p}_{j}^{\top}(t)=\frac{\lambda_{j-1}}{\nu} \underline{p}_{j-1}^{\top}(0)+\underline{p}_{j}^{\top}(k) \underline{\underline{\hat{a}}}_{U: D}(j)+\frac{\mu_{j+1}}{\nu} \underline{p}_{j+1}^{\top}(0) & j=1,2, \cdots, J-1, \\
\underline{p}_{J}^{\top}(t)=\frac{\lambda_{J-1}}{\nu} \underline{p}_{J-1}^{\top}(0)+\underline{p}_{J}^{\top}(0) \underline{\underline{\hat{a}}}_{U: D}(J) & j=J .
\end{array}\right.
$$

Consequently, for each $i \in \mathcal{N}$, we can evaluate the probability $\tilde{p}_{i}(t)$ that $U_{t}=i$ given an initial state probability vector $\underline{p}^{\top}(0)$ by

$$
\tilde{p}_{i}(t)=\sum_{j \in \mathcal{J}} p_{(i, j)}(t) .
$$

\section{Computational Assessment of Volatility Smile for European Call Options Defined on $S_{t}$}

Based on $\left[\tilde{p}_{i}(t)\right]_{i \in \mathcal{N}}$ given in (5.13), it is now possible to evaluate the price $\hat{C}(K, T)$ of an European call option defined on $S_{t} \in e^{R_{U}}$ for $0 \leq t \leq T$ with strike price $K$ and maturity time $T$ as

$$
\hat{C}(K, T)=e^{-r T} E\left[\left\{S_{T}-K\right\}^{+}\right]=e^{-r T} \sum_{i=0}^{N}\left\{e^{u_{i}}-K\right\}^{+} \tilde{p}_{i}(T)
$$

where $\{a\}^{+}=\max \{a, 0\}$. Substituting this price of (6.1) into the Black-Scholes formula, one sees that

$$
\hat{C}(K, T)=S_{0} \Phi\left(\frac{\log \frac{S_{0}}{K}+\left(r+\frac{\hat{\sigma}^{2}(K, T)}{2}\right) T}{\hat{\sigma}(K, T) \sqrt{T}}\right)-e^{-r T} K \Phi\left(\frac{\log \frac{S_{0}}{K}+\left(r-\frac{\hat{\sigma}^{2}(K, T)}{2}\right) T}{\hat{\sigma}(K, T) \sqrt{T}}\right),
$$

where $\Phi(x)=\frac{1}{\sqrt{2 \pi}} \int_{-\infty}^{x} \exp \left(-\frac{y^{2}}{2}\right) d y$. Of particular interest is the implied volatility obtained by solving (6.2) for $\hat{\sigma}(K, T)$.

In what follows, we develop an algorithmic procedure for evaluating $\hat{\sigma}(K, T)$ as a function of $K$ and $T$. For notational convenience, the following functions are introduced:

$$
h^{+}(\sigma) \stackrel{\text { def }}{=} \frac{\log \frac{S_{0}}{K}+\left(r+\frac{\sigma^{2}}{2}\right) T}{\sigma \sqrt{T}} ; \quad h^{-}(\sigma) \stackrel{\text { def }}{=} h^{+}(\sigma)-\sigma \sqrt{T},
$$

and

$$
g(\sigma) \stackrel{\text { def }}{=} S_{0} \Phi\left(h^{+}(\sigma)\right)-e^{-r T} K \Phi\left(h^{+}(\sigma)-\sigma \sqrt{T}\right) .
$$



Algorithm 6.1 (Implied Volatility of the European Call Option)
Input :
$\triangleright r$ : interest rate
$\triangleright K:$ strike price of the option
$\triangleright T$ : maturity time of the option
$\triangleright\left[u_{i}\right]_{i \in \mathcal{N}}$ : discrete state space of $B_{t}^{U}$
$\triangleright\left[\sigma^{-}, \sigma^{+}\right]$: the range of implied volatility satisfying $g\left(\sigma^{-}\right)<\hat{C}(K, T)<g\left(\sigma^{+}\right)$.
$\triangleright \epsilon$ : parameter for stopping the search of implied volatility
Output:
$\triangleleft \hat{\sigma}(K, T)$ : Implied Volatility

Procedure:

1. Compute $\left[\tilde{p}_{i}(T)\right]_{i \in \mathcal{N}}$ and $\hat{C}(K, T)$ based on (5.13)and (6.1) respectively.

2. Loop: Let $\sigma \leftarrow \frac{\sigma^{-}+\sigma^{+}}{2}$ and calculate $g(\sigma)$ by (6.4).

3. If $g(\sigma)<\hat{C}(K, T)$, set $\sigma^{-} \leftarrow \sigma$ and $g\left(\sigma^{-}\right) \leftarrow g(\sigma)$; otherwise, set $\sigma^{+} \leftarrow \sigma$ and $g\left(\sigma^{+}\right) \leftarrow g(\sigma)$.

4. Calculate $\left|g\left(\sigma^{+}\right)-g\left(\sigma^{-}\right)\right|$. If $\left|g\left(\sigma^{+}\right)-g\left(\sigma^{-}\right)\right|<\epsilon$, set $\hat{\sigma}(K, T) \leftarrow \sigma$ and stop; otherwise go to 2 .

Numerical results for $\hat{C}(K, T)$ and $\hat{\sigma}(K, T)$ are plotted in Figures 6.1 and 6.2 respectively, where the parameters are set as $r=0.1, \sigma=1, a=0.09, b=0.1$ and $\delta=0.8$ with initial values $S_{0}=s_{0}=100$ and $V_{0}=0.05$. The range for the strike price is taken to be $60 \leq K \leq 200$ and the range for the maturity time is $1 \leq T \leq 2.5$. The discrete state space of $S_{t}$ is set to be $\left[e^{u_{i}}\right]_{i \in \mathcal{N}}=[20,30, \cdots, 490,500]$ and that of $V_{t}$ is $\left[v_{j}\right]_{j \in \mathcal{J}}=[0.05,0.1, \cdots, 2.0]$. It should be noted that the well-known smile curve can be observed along the $K$-axis, and the smile curve rises monotonically as $T$ increases.

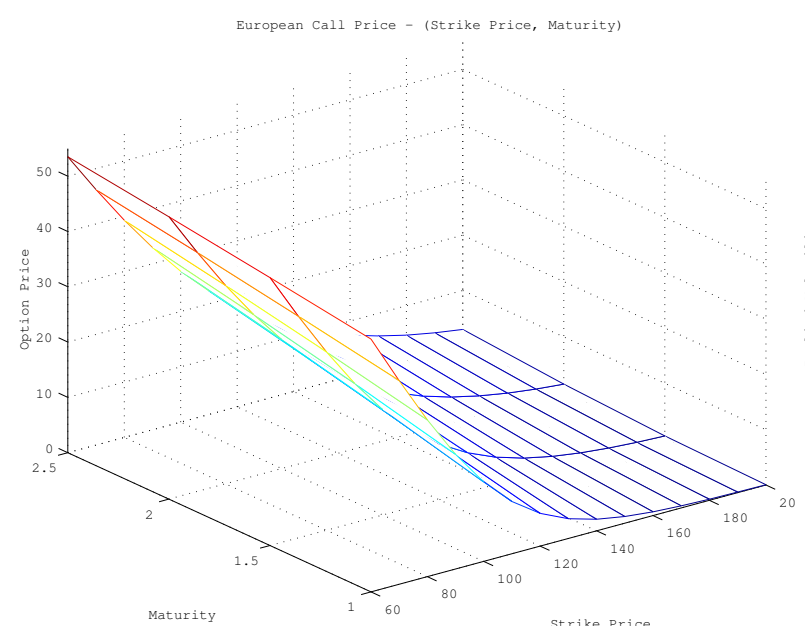

Figure 6.1: Price of European Call Option

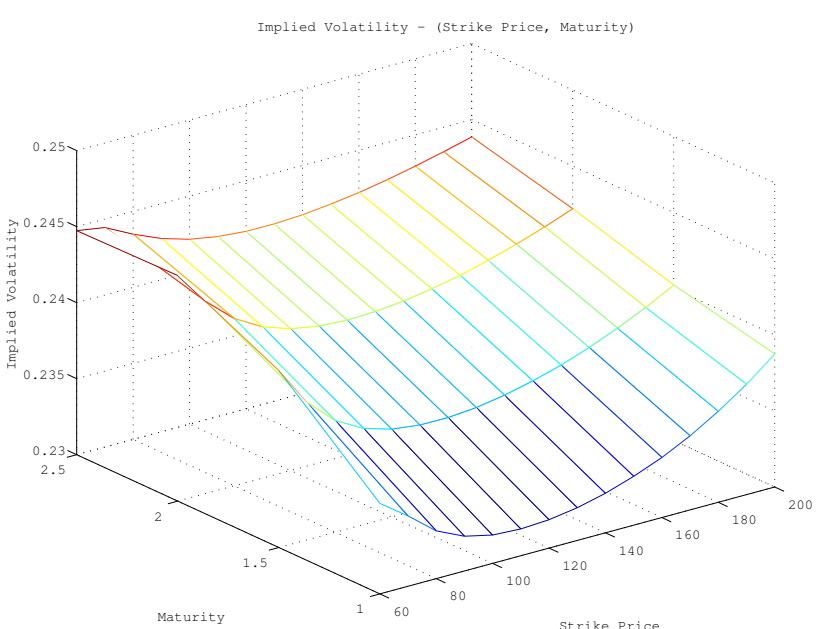

Figure 6.2: Volatility Smile of European Call 
Also of interest are the $\Delta$ and $\Gamma$ of the European call options defined as

$$
\frac{\partial \hat{C}(K, T)}{\partial S_{0}} \approx \frac{\left.\hat{C}(K, T)\right|_{s_{0}+\Delta s_{0}}-\left.\hat{C}(K, T)\right|_{s_{0}}}{\Delta s_{0}}
$$

and

$$
\frac{\partial^{2} \hat{C}(K, T)}{\partial S_{0}^{2}} \approx \frac{\left.\hat{C}(K, T)\right|_{s_{0}+\Delta s_{0}}+\left.\hat{C}(K, T)\right|_{s_{0}-\Delta s_{0}}-\left.2 \hat{C}(K, T)\right|_{s_{0}}}{\left(\Delta s_{0}\right)^{2}},
$$

respectively. These values are plotted as functions of $K$ in Figures 6.3 and 6.4.

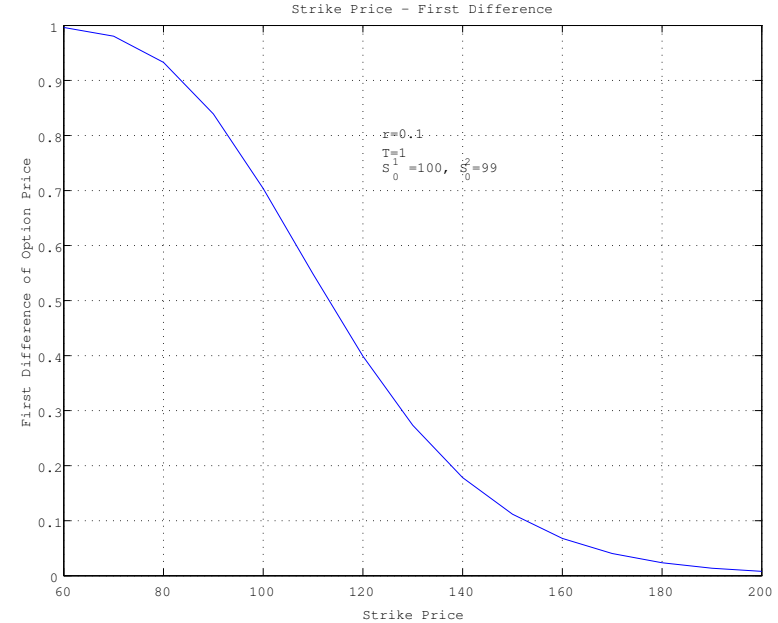

Figure 6.3: Delta of European Call

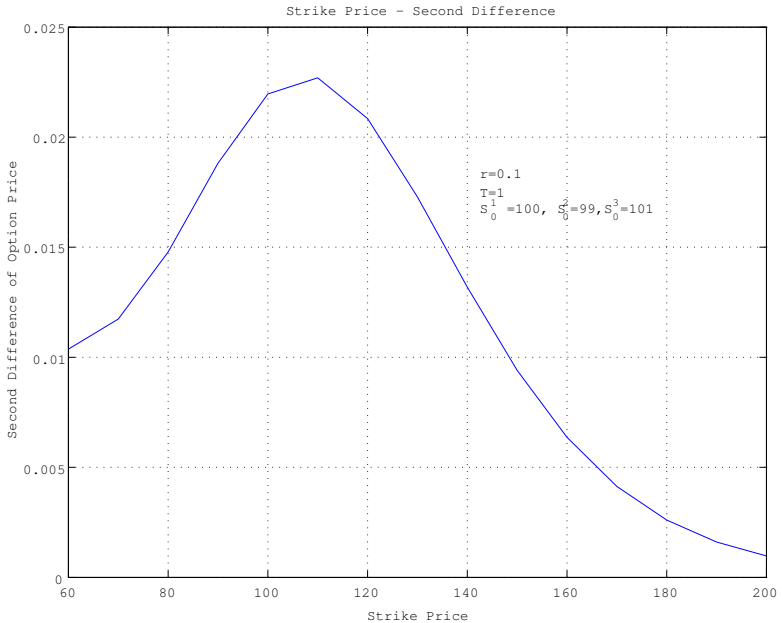

Figure 6.4: Gamma of European Call

\section{Down-and-Out Call Option Pricing}

We now turn our attention to how to evaluate the prices of exotic options defined on $S_{t}$. As a representative of the exotic options, we consider a down-and-out call option maturing at time $T$ with strike price $K$ and a lower barrier level $H<K$. Under the down-and-out call option, starting from $s_{0}=e^{u_{0}}>H$, the option right would be nullified if $S_{t}$ reaches the level $H$ or below by time $T$. Otherwise the option functions as a plain Vanilla European call option having the strike price $K$ at time $T$. Then the price of the down-and-out call option at time $t=0$, denoted by $\pi_{\mathrm{KO}}(0 \mid T)$, can be expressed in terms of the first passage time $T_{u_{0}, \log H}=\inf \left\{t: U_{t}=\log H \mid U_{0}=u_{0}\right\}$ as

$$
\pi_{\mathrm{KO}}(0 \mid T)=e^{-r T} \mathrm{E}\left[\left\{S_{T}-K\right\}^{+} 1_{\left\{T_{u_{0}, \log H}>T\right\}}\right],
$$

where

$$
1_{\{A\}}= \begin{cases}1, & \text { if } A \text { is true } \\ 0, & \text { otherwise. }\end{cases}
$$

Evaluating $\pi_{\mathrm{KO}}(0 \mid T)$ requires the joint distribution of $P\left[U_{t} \leq x, T_{u_{0}, \log H}>T \mid U_{0}=u_{0}\right]$. Since $U_{t}$ is approximated by $B_{t}^{U}$, from the state conversion between $\left\{U_{t}: t \geq 0\right\}$ and $\left\{B_{t}^{U}: t \geq 0\right\}$, the condition $U_{t} \geq \log H, 0 \leq t \leq T$ can be written as

$$
B_{t}^{U} \geq i_{H}, \quad 0 \leq t \leq T,
$$


where $i_{H}=\min \left\{i \in \mathcal{N} \mid u_{i} \geq \log H\right\}$. With little alteration, the numerical approach for computing (5.11) can be employed by making $\left(i_{H}, j\right)$ absorbing for all $j \in \mathcal{J}$. The corresponding transition probability vector $\underline{p}^{* \top}(t)=\left[\underline{p}_{0}^{* \top}(t), \cdots, \underline{p}_{j}^{* \top}(t), \cdots, \underline{p}_{J}^{* \top}(t)\right]$ can then be obtained accordingly, which in turn leads to

$$
p_{i}^{*}(T)=\sum_{j \in \mathcal{N}} p_{(i, j)}^{*}(T) \text { for } i \in\left\{i_{H}, \cdots, N\right\} .
$$

Then from Equation (7.1), the down-and-out call option price can be obtained as

$$
\pi_{\mathrm{KO}}(0 \mid T)=e^{-r T} \sum_{i=i_{H}}^{N}\left\{e^{u_{i}}-K\right\}^{+} p_{i}^{*}(T) .
$$

In order to demonstrate the above numerical procedure, the following down-and-out call option is considered where the strike price is $K=100$ and the barrier price is $H=90$. Other parameters are set similarly as those for Figures 6.1 through 6.4. When $V_{t}$ is constant with $V_{t}=V$, the Black-Scholes formula for the price $B S_{K O}$ of the down-and-out call option is available, where $B S_{K O}$ is given by

$$
\begin{aligned}
B S_{K O}= & {\left[S_{0} \Phi(d)-e^{-r T} K \Phi(d-\tilde{\sigma} \sqrt{T})\right] } \\
& -\left[S_{0}\left(\frac{S_{0}}{H}\right)^{-1-2 r / \tilde{\sigma}^{2}} \Phi(\eta)-e^{-r T} K\left(\frac{S_{0}}{H}\right)^{1-2 r / \tilde{\sigma}^{2}} \Phi(\eta-\tilde{\sigma} \sqrt{T})\right] .
\end{aligned}
$$

Here, one has

$$
\left\{\begin{array}{l}
d=\frac{\log \frac{S_{0}}{K}+\left(r+\frac{\tilde{\sigma}^{2}}{2}\right) T}{\tilde{\sigma} \sqrt{T}}, \\
\eta=\frac{\log \frac{H^{2}}{S_{0} K}+\left(r+\frac{\tilde{\sigma}^{2}}{2}\right) T}{\tilde{\sigma} \sqrt{T}},
\end{array}\right.
$$

and $\tilde{\sigma}=\sigma \sqrt{V}$. In the second and third columns of Table 7.1, the prices $F V_{K O}$ of the downand-out call option with $V_{t}=V$ calculated by our approach are compared with $B S_{K O}$ for $V=0.05$ and $\sigma=0.95,1.00,1.05$, demonstrating fair accuracy of the proposed algorithms. For $V_{t}$ being a square-root process, no analytical results are available to evaluate the price $S V_{K O}$ of the down-and-out call option. However, the computational algorithms proposed in this paper enables one to evaluate $S V_{K O}$ for a variety of parameter values with speed. These prices are given in the fourth column of Table 7.1 .

Table 7.1 Comparison of Option Prices $\left(S_{0}=100, K=100, H=90\right)$

\begin{tabular}{|c|c|c|c|}
\hline$\sigma$ & $B S_{K O}$ & $F V_{K O}$ & $S V_{K O}$ \\
\hline 0.95 & 11.2236 & 11.2725 & 11.6912 \\
\hline 1.00 & 11.2449 & 11.2971 & 11.6987 \\
\hline 1.05 & 11.2591 & 11.3143 & 11.7005 \\
\hline
\end{tabular}

The prices of other exotic options can be computed in a similar manner, provided that such prices are expressed in terms of the joint distribution of $\left\{S_{t}, V_{t}\right\}$ and some of the associated first passage times. 


\section{References}

[1] Albanese, C. And Kuznetsov, A.(2005). Discretization Schemes for Subordinated Processes, Available at http://www.math.toronto.edu/albanese.

[2] F. Black and M. Scholes(1973). The Pricing of Options and Corporate Liabilities, Journal of Political Economy, 81, 637-659.

[3] J.C.Cox, J.E.Ingersoll, And S.A.Ross(1985). An Intertemporal General Equilibrium Model of Assete Prices, Econometrica, 53, 363-384.

[4] Gotoh, J., Jin, H. And Sumita, U.(2006). Numerical Evaluation of Dynamic Behavior of Ornstein-Uhlenbeck Processes Modified by Vairous Boundaries and Its Application to Pricing Barrier Options. Discussion paper series, No.1149, Depratment of Social Systems and Management, University of Tsukuba, 2006, submitted for publication. Available at http://www.sk.tsukuba.ac.jp/SSM/libraries/list1126.html

[5] Gunter, W., Thomas, A. And Uwe, W.(2001). Valuation of Options in Heston's Stochastic Volatility Model Using Finite Element Methods, Foreign Exchange Risk, Risk Publications, London.

[6] Heston, S.(1993). A Closed-form Solution for Options with Stochastic Volatility with Application to Bond and Currency Options, Review of Financial Studies, 6, 327-343.

[7] Hull, J. And White, A. (1987). The Pricing of Options on Assets with Stochastic Volatilities, Journal of Finance, 42, 281-300

[8] Karlin, S. And McGregor, J. L.(1958). Linear Growth, Birth and Death Process, Journal of Mathematics and Mechanics 7,634-662.

[9] KeIlson, J.(1979). Markov Chain Models: Rarity and Exponentiality, Applied Mathematical Science Series, 28, Springer, New York.

[10] Lamberton, D. And Lapeyre, B.(1997). Introduction to Stochastic Calculus Applied to Finance, Chapman and Hall.

[11] Stein, E. M. And Stein, J. C.(1997). Stock Price Distributions with Stochastic Volatility: An Analytic Approach, Review of Financial Studies, 4, 727-752.

[12] Sumita ,U.(1981). Development of The Laguerre Transform Method for Numerical Exploration of Applied Probability Methods, Doctoral thesis. Graduate school of Management, University of Rochester.

[13] Sumita, U. And KiJima, M.(1988). Theory and Algorithms of the Laguerre Transform, Part 1 : Theory, Journal of the Operations Research Society of Japan 31, 467-494.

[14] Sumita, U. And KiJima, M.(1991). Theory and Algorithms of the Laguerre Transform, Part 2 : Algorithm, Journal of the Operations Research Society of Japan 34, 449-477.

[15] Schoutens, W.(2000). Stochastic processes and orthogonal polynomials, Springer, New York. 